\title{
A comprehensive analysis of the expression, epigenetic and genetic changes of HNF1B and ECI2 in 122 cases of high-grade serous ovarian carcinoma
}

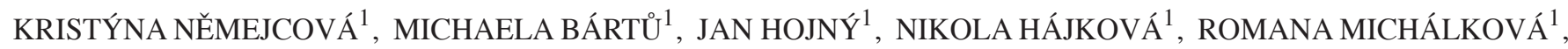 \\ EVA KRKAVCOVÁ ${ }^{1}$, IVANA STRUŽINSKÁ ${ }^{1}$, HIEP QUANG BUI ${ }^{1}$, PAVEL DUNDR ${ }^{1}$, \\ DAVID CIBULA ${ }^{2}$ and KATEŘINA JIRSOVÁ ${ }^{3}$ \\ ${ }^{1}$ Institute of Pathology, ${ }^{2}$ Gynecologic Oncology Center, Department of Obstetrics and Gynecology, \\ ${ }^{3}$ Institute of Biology and Medical Genetics, First Faculty of Medicine, \\ Charles University and General University Hospital in Prague, 12800 Prague, Czech Republic
}

Received July 29, 2020; Accepted December 3, 2020

DOI: $10.3892 / \mathrm{ol} .2021 .12446$

\begin{abstract}
High-grade serous ovarian cancer (HGSC) is the most common subtype of ovarian cancer, with a poor prognosis; however, most studies concerning ovarian carcinoma have focused mainly on clear cell carcinoma. The involvement of hepatocyte nuclear factor $1 \beta$ (HNF1B) in the carcinogenesis of HGSC has not yet been fully elucidated. To the best of our knowledge, the present study is the first to analyse the expression of the possible downstream target of HNF1B, enoyl-CoA $(\Delta)$ isomerase 2 (ECI2), in HGSC. The present study performed a comprehensive analysis of HNF1B mRNA and protein expression, and epigenetic and genetic changes, as well as an analysis of ECI2 mRNA and protein expression in 122 cases of HGSC. HNF1B protein expression was detected in 28/122 cases, and was positively associated with lymphovascular invasion $(\mathrm{P}=0.025)$. Protein expression of ECI 2 was detected in 115/122 cases, but no associations with clinicopathological variables were revealed. Therefore, ECI2 does not seem to function as a suitable prognostic marker for HGSC. In the sample set, a positive correlation between HNF1B and ECI2 protein expression was detected $(\mathrm{P}=0.005)$. HNF1B mRNA was also positively correlated with HNF1B protein expression $(\mathrm{P}=0.001) . H N F 1 B$ promoter methylation was detected in 26/67 (38.8\%) of cases. A novel pathogenic somatic HNF1B mutation was detected in $1 / 61(1.6 \%)$ of the analysed HGSC
\end{abstract}

Correspondence to: Dr Kristýna Němejcová, Institute of Pathology, First Faculty of Medicine, Charles University and General University Hospital in Prague, Studnickova 2, 12800 Prague 2, Czech Republic

E-mail: kristyna.nemejcova@vfn.cz

Key words: ovarian carcinoma, high-grade serous carcinoma, hepatocyte nuclear factor $1 \beta$, enoyl-CoA $(\Delta)$ isomerase 2, immunohistochemistry, mutation analysis, methylation, mRNA cases. No other correlations between the examined SNPs (rs4430796, rs757210 and rs7405776), HNF1B promoter methylation, HNF1B/ECI2 expression or clinicopathological characteristics were found.

\section{Introduction}

Hepatocyte nuclear factor $1 \beta(H N F 1 B)$ is a transcription factor which plays a crucial role during embryonic development and differentiation of various organs (liver, kidney, lung, gonads, biliary system, and pancreas) (1). The HNF1B protein regulates the expression of multiple genes involved in cell cycle modulation, susceptibility to apoptosis, and response to oxidative stress $(2,3)$. A growing number of studies have demonstrated the potential involvement of HNF1B in the tumorigenesis of some solid tumours. However, the precise mechanism by which HNF1B influences tumour development has not yet been fully elucidated. Recent findings suggest that HNF1B may act either as a tumour suppressor or an oncogene in different cancers, depending on the type of tissue and the tumour (4). Some authors regard HNF1B as a pro-differentiation factor with a suppressive influence on epithelial-to-mesenchymal transition (EMT) in unmethylated healthy tissues (5). However, others have described its role as an epithelial-specific oncogene, which induces a cancerous phenotype, epithelial-to-mesenchymal transition, and invasive behaviour (6).

To date, the only studies concerned with the involvement of the $H N F 1 B$ gene in the carcinogenesis of tumours of the female genital tract are mostly genome-wide associated studies (GWAS), exploring the influence of single nucleotide polymorphisms (SNPs) on ovarian and endometrial cancers. It has been reported that in endometrial cancer there is an association between the SNP rs4430796 and the risk and prognosis of the disease (7-11). In ovarian cancer, several SNPs of interest have been identified. The SNP rs757210 was found to be associated with promoter methylation in high-grade serous carcinoma (HGSC), and its involvement 
also reached borderline significance in ovarian clear cell carcinoma (OCCC) (5). There are other SNPs which have also been implicated in influencing promotor methylation: SNP rs7405776 is associated with increased methylation in HGSC, and SNP rs11651755 was reported to be associated with unmethylated status and an increased HNF1B expression in OCCC (10).

The immunohistochemical expression of HNF1B was initially considered to be a highly specific marker of OCCC (7). However, recent studies have also described the positivity of HNF1B in some other malignant and benign tumours of the female genital tract, and even in non-neoplastic endometrial lesions and normal endometrium (12-14). These findings demonstrate that the specificity of HNF1B is less than was originally assumed. However, if we take into account the staining intensity, then the characteristic diffuse strong nuclear expression has been found predominantly in clear cell carcinomas $(15,16)$.

Concerning the downstream targets, the transcription factor HNF1B seems to be involved in several key regulatory pathways including cell cycle regulation, epithelial-mesenchymal transition, cell migration, adhesion, and proliferation. One of the possible downstream targets of HNF1B seems to be enoyl-CoA- $(\Delta)$ isomerase 2 (ECI2), which belongs to the acyl-CoA-binding domain (ACBD) family (17). The ECI2 enzyme plays a role in glucose and lipid metabolism, allowing for the re-entry of the enoyl-CoA into the $\beta$-oxidation cycle $(18,19)$. Currently, the exact role of ECI2 in carcinogenesis is still poorly understood. However, a recent study has shown that the inhibition of expression of ECI2 in prostate cancer leads to decreased glucose utilization, the accumulation of fatty acids, and the down-regulation of the cell cycle-associated genes (19). Furthermore, the regulatory role of HNF1B in ECI2 expression was investigated on a mouse model of prostate cancer (17). The authors found that the increased expression levels of HNF1B in the initial stages of prostatic cancer play a tumour-protective role and are associated with ECI2 upregulation. Nevertheless, the precise mechanism explaining the possible interactions between HNF1B and ECI2 and their role in tumorigenesis have not yet been fully elucidated. ECI2 is targeted to mitochondria and peroxisomes, and probably mediates their mutual interaction (20). The data about the expression of ECI2 in ovarian cancer is currently unknown.

This is the first retrospective analysis of a large subset of HGSC which includes a complex genetic, epigenetic, and histochemical analysis of HNF1B, complete with an analysis of the possible HNF1B's downstream target ECI2. The goals of our study were: i) To perform a comprehensive molecular and immunohistochemical analysis of HNF1B in HGSC, including the protein and mRNA expression, and the epigenetic and genetic changes of HNF1B, ii) to perform a protein and mRNA expression analysis of its downstream target ECI2, iii) to correlate HNF1B methylation with mRNA or protein expression, and to study the association of HNF1B and ECI2 expression, iv) to analyse the relationship between the molecular and immunohistochemical patterns with the clinicopathological variables and clinical outcomes.

\section{Materials and methods}

Samples. Formalin-fixed paraffin-embedded (FFPE) tissue blocks were primarily used for the analyses. Where available, the corresponding fresh-frozen tissue (FT) extracted from the same individuals was used for the subsequent molecular DNA/RNA analysis. The FFPE samples were obtained from the archive files of the Institute of Pathology, and the FT samples were provided by the Bank of Biological Material (BBM) of the First Faculty of Medicine, Charles University in Prague. The FFPE samples were stored in the archives at $10-14^{\circ} \mathrm{C}$ and the FT samples were stored in the RNAlater stabilization solution (Qiagen) at $-80^{\circ} \mathrm{C}$, according to the manufacturer's protocol (Stabilization of RNA in Harvested Animal Tissues; Qiagen).

In total, 122 FFPE samples of HGSC were selected for immunohistochemical analysis, including 69 cases with an available FT for subsequent epigenetic, genetic, and expression analysis. All FFPE and FT tissue samples were reviewed by senior pathologists who selected the eligible areas for tumour analysis. The mean age of patients was 59 years (median, 60 years; range, 36-81 years). The clinicopathological characteristics of the samples analysed are summarized in Tables I and II.

Immunohistochemical analysis. The FFPE blocks were used for the construction of the tissue microarrays (TMAs). Two cores (each $2.0 \mathrm{~mm}$ in diameter) were taken from a single donor block from each case, using the tissue microarray instrument TMA Master (3DHISTECH Ltd.). All samples of FFPE tissue were sectioned at a thickness of 4-5 $\mu \mathrm{m}$, and the immunohistochemical (IHC) analysis was performed using an antibody against HNF1B (polyclonal, dilution 1:500; Sigma-Aldrich; Merck KGaA), and a rabbit antibody against ECI2 (polyclonal, dilution 1:100, product no. ab235322; Abcam) in the automated staining instrument Ventana BenchMark ULTRA (Roche). Heat induced antigen retrieval, including pre-treatment, was carried out in a citrate buffer ( $\mathrm{pH}$ 6.0). For visualization, the OptiView DAB IHC Detection Kit (Ventana, Roche) was used. For HNF1B, only nuclear staining was regarded as positive, and for ECI 2 only the cytoplasmic staining was evaluated. The expression of both markers was double-blindly evaluated by two pathologists.

The immunohistochemical results were assessed semi-quantitatively, using the $\mathrm{H}$-score method described previously by others (21). The H-score combines the percentage of positive cells and the level of staining intensity (1+ for weak, $2+$ for moderate, and $3+$ for strong intensity). The final $\mathrm{H}$-score is then calculated according to the following formula: $[1 \mathrm{x}(\%$ of cells $1+)+2 x(\%$ of cells $2+)+3 x(\%$ of cells $3+)$ ], with the results ranging from 0 to 300 .

DNA and RNA isolation, quality control and cDNA synthesis. Firstly, the FT tissues were thawed and homogenized (10-30 mg) in the presence of $600 \mu \mathrm{l}$ of RLT Buffer (Qiagen) with $6 \mu 1$ of 14.3M 2-mercaptoehthanol (Sigma-Aldrich; Merck KGaA) using MagNA Lyser Green Beads tubes in MagNA Lyser Instrument (Roche), as described in Bartu et al (22).

The total DNAs and RNAs were isolated according to the Simultaneous Purification of Genomic DNA and Total RNA 
from Animal Tissues protocol using an AllPrep DNA/RNA Mini kit (Qiagen). The isolated DNA and RNA samples were quantified by the NanoDrop 2000 (Thermo Fisher Scientific, Inc.).

The RNA Quality Number (RQN) of the isolated total RNA was determined using the Fragment Analyzer (AATI) capillary electrophoresis system and Standard RNA kit (AATI). Those RNA samples with an RQN lower than 7.5 were removed from further analysis (tissue samples RQN mean=9.3; range 5-10). Otherwise, $3.75 \mu \mathrm{g}$ of total RNA of each sample (where available) was treated by DNase I (Thermo Fisher Scientific, Inc.), and cDNA was synthetized in a $40 \mu 1$ reaction using SuperScript III Reverse Transcriptase (Thermo Fisher Scientific, Inc.) with random hexamers (Roche) as described in Dundr et al (23).

HNF1B mutation analysis. The mutation analysis of the HNF1B gene included the analysis of all the coding region (exons 1-9, RefSeq NM_000458.2) with adjacent intronic sequences $( \pm 15 \mathrm{bp})$ and two deep intronic regions containing the rs7527210 and rs4430796 polymorphisms. The FT samples were analysed by amplicon next generation sequencing, as described previously (22).

Primers for the analysis of rs7405776 were designed (rs7405776_Forward: agccacagactctagatctgg, rs7405776_ Reverse: caaagtgctgggattataagtgtg), and the amplicons were sequenced by Sanger sequencing on the ABI3500 Genetic Analyser (Thermo Fisher Scientific, Inc.).

Mutations which are not found in the literature, the Single Nucleotide Polymorphism Database (dbSNP, http://www.ncbi. nlm.nih.gov/SNP/), the ClinVar Database (https://www.ncbi. nlm.nih.gov/clinvar/), or in the Catalogue of Somatic Mutations in Cancer (COSMIC, http://www.sanger.ac.uk/cosmic; databases accessed September 2020) are considered as novel.

HNF1B promoter methylation analysis. The epigenetic analysis of the HNF1B promoter region was performed as described previously (22). The isolated DNA was converted using the EZ DNA Methylation-Lightning Kit (Zymo Research) according to the manufacturer's instructions. The primers for the PCR amplification of both the methylated and unmethylated alleles were designed using Methprimer software (http://www. urogene.org/cgi-bin/methprimer/methprimer.cgi;): HNF1B_ met_forward_TTTTTGGATTTGTTAAGTTAGTGTTTT, HNF1B_met_reverse CCCTTCCTAAATAATCAATTTCTC TT (PCR product chr17:36105251-36105506, GrCh37). The PCR amplification was carried out using the following protocol: $95^{\circ} \mathrm{C} \_12 \mathrm{~min}, 40 \mathrm{x}\left(95^{\circ} \mathrm{C} \_15 \mathrm{sec}, 58^{\circ} \mathrm{C} \_30 \mathrm{sec}, 72^{\circ} \mathrm{C} \_30 \mathrm{sec}\right)$, $72^{\circ} \mathrm{C} \_5 \mathrm{~min}$, and followed by melting curve analysis in the LightCycler 480 II instrument (Roche). The amplification and melting curves were analysed by the LightCycler 480 II Software, and then compared to the control mixes.

Analysis of $m R N A$ expression. The expression analysis was performed by using cDNA samples and the droplet digital PCR system (ddPCR; Bio-Rad). All the ddPCR steps, including the expression of three potential reference mRNA targets (POLR2A, HPRT1 and ATP5F1B), two HNF1B mRNA targets (in 5' and 3'UTR), and one ECI2 target, as well as repeatability and reproducibility, were optimized prior to the general ddPCR analysis.
The ddPCR reactions were prepared according to the manufacturer's instructions using the QX200 ddPCR EvaGreen Supermix (Bio-Rad), $1 \mu 1$ of cDNA template (which corresponds to approximately $90 \mathrm{ng}$ of total RNA) and 4 pmol of each of the primer pairs $(200 \mathrm{nM}$ final concentration) in $20 \mu \mathrm{l}$ of reaction volume. Master mix droplets were generated by the QX200 AutoDG instrument (Bio-Rad), and the samples were amplified by a $5 \mathrm{~min}$ incubation at $95^{\circ} \mathrm{C}$, then 40 cycles of $95^{\circ} \mathrm{C}$ for $30 \mathrm{sec}$ and $58^{\circ} \mathrm{C}$ for $1 \mathrm{~min}$, followed by the final signal stabilization steps consisting of $4^{\circ} \mathrm{C}$ for $5 \mathrm{~min}$, finishing with $90^{\circ} \mathrm{C}$ for $5 \mathrm{~min}$. The resulting data was acquired using the QX200 Droplet Reader instrument (Bio-Rad) with the standard acquisition protocol for the EvaGreen master mix and analysed by QuantaSoft software (Bio-Rad). The threshold for positive droplet signals of each of the three final amplicons (reference POLR2A; HNF1B 3'UTR and ECI2 targets) was set as the average value of the thresholds, which were calculated automatically by the QuantaSoft software during the optimization steps. The thresholds of all the acquired targets were manually confirmed. The final data of the targets (HNF1B and ECI2), expressed as the number of templates in $20 \mu \mathrm{l}$ of the master mix (which corresponds to $1 \mu \mathrm{l}$ of cDNA), were re-calculated to the number of targets per one thousand reference POLR2A targets, and analysed as described below in the Statistical analysis section. Only samples with a positive HNF1B mRNA expression (the reliable limit for positivity was set to more than 50 copies/ $\mu 1$ of cDNA) were further compared to the mRNA ECI2 expression.

Statistical analysis. All the statistical analyses were performed using the software Statistica (TIBCO). The Shapiro-Wilk test was used to control data normality. With respect to the non-normal data distribution, non-parametric analyses were conducted (Mann-Whitney U test or Kruskal-Wallis H-test) in order to analyse the association between HNF1B or ECI2 protein expression (H-score) and the clinico-pathological variables (age at the time of diagnosis, FIGO stage, lymphovascular invasion (LVSI), neoadjuvant therapy, local and distant recurrence, and, in the case of HNF1B, promoter methylation status and the presence of the three analysed SNPs: rs4430796, rs757210, rs7405776. For the evaluation of the effect of independent clinicopathological characteristics on the categorized $\mathrm{H}$-score or methylation status, the Pearson $\chi^{2}$ test or the Fisher's exact test was used, depending on the expected frequencies (24). Correlations between two continuous variables were analysed using Pearson's method.

For the purposes of $\chi^{2}$ tests and survival analyses, the $\mathrm{H}$-score of both HNF1B and ECI2 was categorized into two groups with respect to their median values (HNF1B: Group 1: H-score 0-19; group 2: H-score 20-300; ECI2: Group 1: H-score 0-99, group 2: H-score 100-300).

Survival analyses were plotted using the Kaplan-Meier model and the differences between curves were tested for significance using the log-rank test. Disease-free survival (DFS), local recurrence-free survival (LFS) and metastasis-free survival (MFS) were defined as the time from the date of the diagnosis to the date of a specific event: Death as a result of the diagnosis (DFS), the first local recurrence (LFS) 
and/or the first distant metastasis (MFS). If the patient did not show any of the monitored events, the case was censored in the analysis at the date of the last follow-up. The probability of survival between the HNF1B and ECI2 H-score group 1 and group 2 was also compared. All tests were two-sided and a $\mathrm{P}$-value of $<0.05$ was considered as significant.

The Cancer Genome Atlas (TCGA) data access and analysis of $H N F 1 B$ promoter methylation and $m R N A$ expression of HNFIB and ECI2. The data from the TCGA, including the clinico-pathological findings and mRNA expression (z-score) of HNF1B and ECI2, was downloaded through the cBioPortal (www.cbioportal.org; (TCGA, Ovarian Serous Cystadenocarcinoma, Firehose Legacy, access June 2020)). Additionally, the HNF1B promoter methylation data was downloaded through the portal https://mexpress.be/ (gene: HNF1B, cancer type: Ovarian serous cystadenocarcinomas, access April 2020) to compare our results. The TCGA sample set included 569 samples of the histological type of serous cystadenocarcinoma with stated stage, neoplasm histologic grade 2 or 3 , and methylation status. The mRNA expression of HNF1B and ECI2 was available only for 178 of these samples. The locus cg12788467 (position to the relative transcription start site -238, GRCh37), which is also included in our analysed promoter region, was used for the investigation of the methylation status of the HNF1B promoter region. The $\beta$-value $>0.3$ of this locus was determined as hypermethylated (25).

\section{Results}

Immunohistochemical findings and clinicopathological associations. The immunohistochemical analysis of both markers (HNF1B and ECI2) was performed in all 122 cases of HGSC. The nuclear protein expression of HNF1B was generally very low (mean=21.8, median=0). The HNF1B positivity was observed in 28 cases (H-score ranging from 20 to 99 in 15 cases, $\mathrm{H}$-score ranging from 100 to 200 in 13 cases). The rest of the samples were negative (H-score $=0, n=112$; Fig. $1 \mathrm{~A})$. The intensity of staining was mostly mild to moderate (Fig. 1B). Focal strong nuclear staining was observed in 17 cases, but the portion of positive cells did not exceed $30 \%$ of all the tumour cells. None of our cases exceeded an H-score value of 200. The cytoplasmic expression of ECI2 ranged from 0 to 190 (mean=107.3, median=105; Fig. 1C). Seven cases did not express ECI2 at all (H-score=0; Fig. 1D). Adjacent non neoplastic ovarian tissue showed positive cytoplasmic staining of ECI2 and nuclear negativity of HNF1B staining in all samples.

A higher protein HNF1B expression was associated with lymphovascular invasion $(\mathrm{Z}=-2.23, \mathrm{~N}=91, \mathrm{P}=0.025$; Table I). No other significant associations of HNF1B or ECI2 protein expression with clinico-pathological characteristics were observed (Tables I and II).

The relationship between HNF1B or ECI2 expression and disease outcome, including disease-free survival (DFS), local recurrence-free survival (LFS), and metastasis-free survival (MFS) was analysed. No significant association between the HNF1B or ECI2 expression (H-score categorized into two groups, as described in Materials and methods) and the probability of DFS $(\mathrm{Z}=1.39, \mathrm{~N}=120, \mathrm{P}=0.164 / \mathrm{Z}=-0.32, \mathrm{~N}=120$, $\mathrm{P}=0.746$, respectively), LFS $(\mathrm{Z}=-0.09, \mathrm{~N}=102, \mathrm{P}=0.922 / \mathrm{Z}=0.32$, $\mathrm{N}=102, \mathrm{P}=0.750$, respectively), or MFS ( $\mathrm{Z}=-0.06, \mathrm{~N}=102$, $\mathrm{P}=0.948 / \mathrm{Z}=-0.11, \mathrm{~N}=102, \mathrm{P}=0.915$, respectively) was found. However, we observed a non-significant trend suggesting an association between a lower HNF1B expression and a higher probability of disease-free survival (Fig. 2).

Relationship between genetic, epigenetic, and expression characteristics. The mutation analysis of HNF1B was successfully performed on $61 \mathrm{FT}$ tumour samples. In one of the 61 (1.6\%) analysed tumour samples, a nonsense somatic mutation NM_000458.2: c.1063C>T, p.(Q355X) with a variant allele frequency $68 \%$ was found. This case showed complete negativity of the HNF1B protein expression $(\mathrm{H}$-score=0), together with moderate positivity of ECI2 (H-score $=100)$.

The $H N F 1 B$ promoter methylation was detected in 26 $(38.8 \%)$ of the 67 analysed samples. The methylation status did not correlate with any of the tested clinicopathological characteristics (Table III). Moreover, none of the three investigated SNPs (rs7405776, rs4430796, and rs7527210) showed any association with HNF1B protein expression (Table I) or HNF1B promoter methylation (Table III). Samples without $H N F 1 B$ promoter methylation had a significantly higher HNF1B mRNA expression $(\mathrm{Z}=2.91, \mathrm{~N}=46, \mathrm{P}=0.003)$. Association of HNF1B promoter methylation with protein expression was marginally non-significant $(\mathrm{Z}=1.91, \mathrm{~N}=67, \mathrm{P}=0.056)$.

Correlations of HNF1B and ECI2 expression on $m R N A$ and protein level. HNF1B expression was successfully analysed in all of the 122 HGSC FFPE samples (protein) and corresponding 47 FT samples (mRNA). ECI2 mRNA expression was analysed in a limited subset of 14/47 (29.8\%) FT samples, where the HNF1B mRNA expression was positive. ECI2 protein expression was successfully analysed and compared in all 122 HGSC samples.

Increased mRNA expression of HNF1B significantly correlated with increased protein expression $\left(\mathrm{F}=12.18, \mathrm{R}^{2}=0.213\right.$, $\mathrm{N}=47, \mathrm{P}=0.001$; Fig. 3 ). A positive correlation was not observed for ECI2 mRNA and protein expression $\left(\mathrm{F}=0.51, \mathrm{R}^{2}=0.041\right.$, $\mathrm{N}=14, \mathrm{P}=0.488$, Fig. 3). A significant positive association was observed when comparing HNF1B with ECI2 protein expression ( $\mathrm{F}=8.03, \mathrm{R}^{2}=0.063, \mathrm{~N}=122, \mathrm{P}=0.005$; Fig. 3), however, no association was observed for mRNA expression $(\mathrm{F}=0.05$, $\mathrm{R}^{2}=0.005, \mathrm{~N}=14, \mathrm{P}=0.816$; Fig. 3 ). However, these results may be influenced by the limited number of samples.

Re-analysis of mRNA and epigenetic status of TCGA dataset. Based on TCGA data, the methylation of the HNF1B promoter was detected in 315/569 (55\%) samples of HGSC. A significantly higher expression of HNF1B mRNA was detected in the non-methylated cases, in comparison to the methylated cases $(\mathrm{Z}=3.32, \mathrm{~N}=71, \mathrm{P}<0.001)$. No statistically significant correlation between the mRNA expression of HNF1B and ECI2 was found $\left(\mathrm{F}=0.25, \mathrm{R}^{2}=0.001, \mathrm{~N}=71, \mathrm{P}=0.614\right)$.

\section{Discussion}

HNF1B is a transcriptional factor implicated in the carcinogenesis of solid tumours, and its role in the tumorigenesis of 

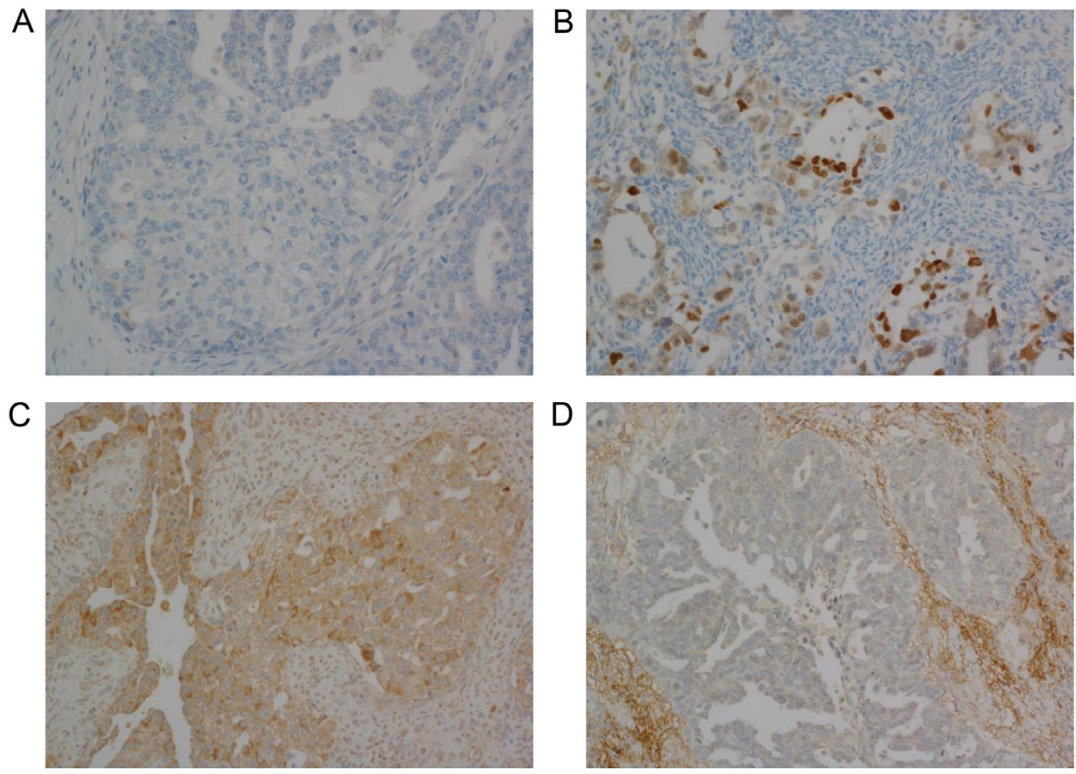

Figure 1. Immunohistochemical staining of HNF1B and ECI2 in HGSC. (A) Complete lack of nuclear expression of HNF1B (x200). (B) Variably intense (mild to strong) nuclear expression of HNF1B (x200). (C) Variably intense (mild to strong) cytoplasmic expression of ECI2 (x200). (D) Complete lack of cytoplasmic expression of ECI2 (x200). HGSC, high-grade serous ovarian cancer; HNF1B, hepatocyte nuclear factor 1 $\beta$; ECI2, enoyl-CoA $(\Delta)$ isomerase 2.
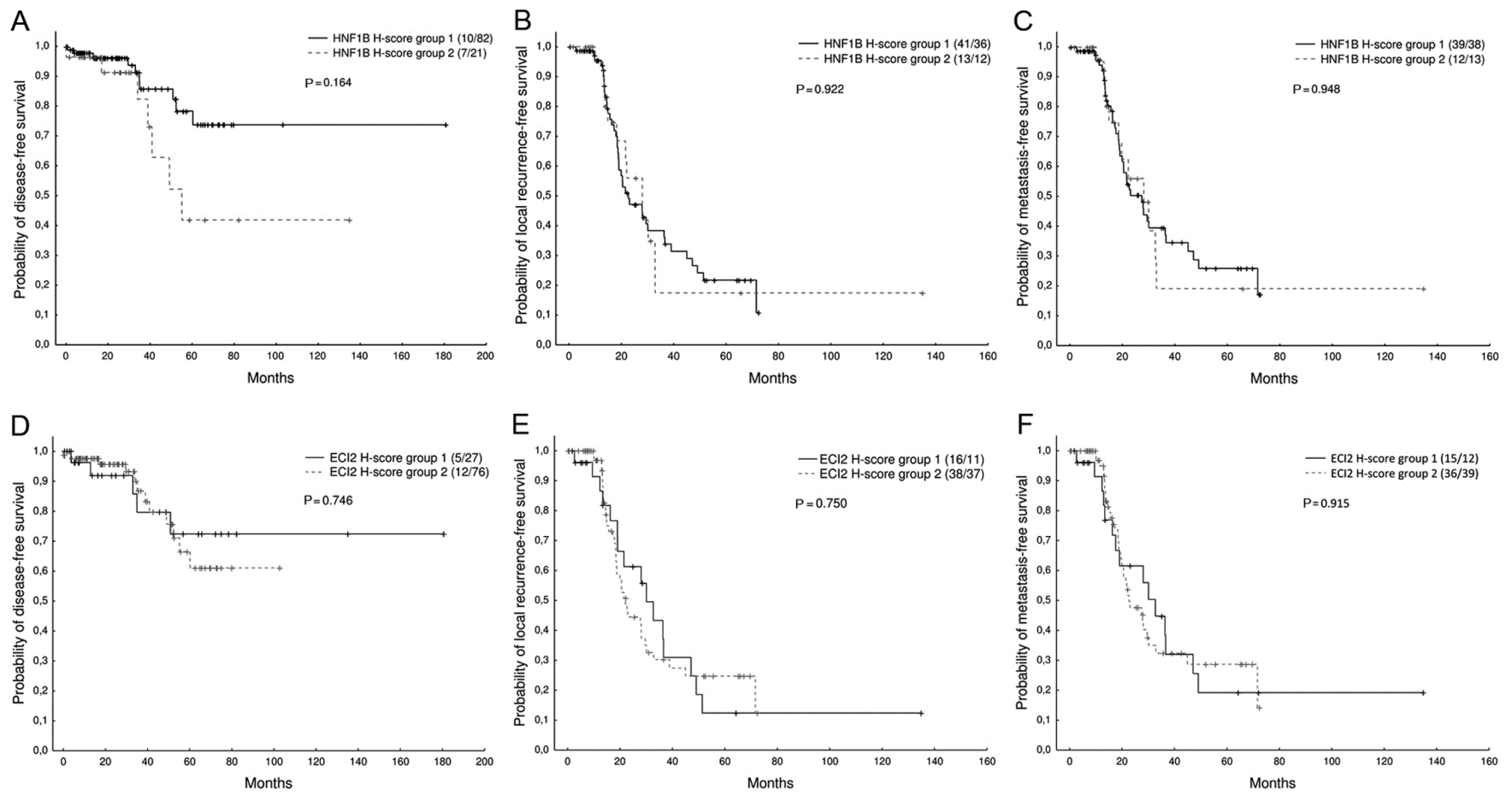

Figure 2. Kaplan-Meier plot of the estimated probability of (A and D) disease-free, (B and E) local recurrence-free and (C and F) metastasis-free survival in relation to H-score of HNF1B/ECI2 (HNF1B: Group 1: H-score 0-19; group 2: H-score 20-300; ECI2: Group 1: H-score 0-99, group 2: H-score 100-300). Censored/complete cases are reported in parentheses. HNF1B, hepatocyte nuclear factor 1 $\beta$; ECI2, enoyl-CoA $(\Delta)$ isomerase 2

the female genital tract has so far mostly focused on clear cell carcinomas, especially OCCC $(7,26,27)$. However, the expression of this marker was also found in HGSC and in other types of ovarian tumours $(28,29)$. HGSC is the most common and most aggressive subtype of ovarian cancer with poor prognosis, accounting for up to $80 \%$ of all deaths from ovarian carcinoma (30). The possible involvement of HNF1B in the carcinogenesis of HGSC has not yet been fully elucidated. In
HGSC, the expression of HNF1B is predominantly low, and the current hypothesis is that in this context HNF1B acts as a tumour suppressor gene $(5,7,26)$.

Some knowledge about this issue can be found in the GWAS, which were mostly focused on the involvement of various SNPs in ovarian cancer. Ross-Adams et al examined the relationship between SNPs and epigenetic changes in ovarian and prostatic cancers, also exploring their 
Table I. Association of HNF1B protein expression (H-score) and clinicopathological characteristics, based on 122 cases of HGSC.

\begin{tabular}{|c|c|c|c|c|c|c|c|}
\hline Characteristic & $\mathrm{N}$ & H-score mean & H-score median & P-value & H-score group 1 & $\mathrm{H}$-score group 2 & P-value \\
\hline $\begin{array}{l}\text { Age of diagnosis }(\text { mean }=59 \text {, } \\
\text { median }=60) \text {, years }\end{array}$ & & & & $0.094^{\mathrm{b}}$ & & & $0.273^{\mathrm{c}}$ \\
\hline$<60$ & 59 & 13.8 & 0 & & 48 & 11 & \\
\hline$\geq 60$ & 63 & 29.3 & 0 & & 46 & 17 & \\
\hline FIGO $^{\mathrm{a}}$ & & & & $0.953^{\mathrm{b}}$ & & & $0.676^{\mathrm{c}}$ \\
\hline I & 10 & 27.6 & 0 & & 7 & 3 & \\
\hline II & 7 & 18.6 & 0 & & 6 & 1 & \\
\hline III & 83 & 22.5 & 0 & & 62 & 21 & \\
\hline IV & 20 & 19.2 & 0 & & 17 & 3 & \\
\hline Lymphovascular invasion $^{\mathrm{a}}$ & & & & $0.025^{\mathrm{b}}$ & & & $0.023^{\mathrm{c}}$ \\
\hline Yes & 63 & 27.7 & 0 & & 45 & 18 & \\
\hline No & 28 & 8.6 & 0 & & 26 & 2 & \\
\hline Neoadjuvant therapy & & & & $0.607^{\mathrm{b}}$ & & & $0.768^{c}$ \\
\hline Yes & 28 & 24.4 & 0 & & 21 & 7 & \\
\hline No & 94 & 20.9 & 0 & & 73 & 21 & \\
\hline Local recurrence ${ }^{\mathrm{a}}$ & & & & $0.261^{\mathrm{b}}$ & & & $0.734^{\mathrm{c}}$ \\
\hline Yes & 55 & 16.8 & 0 & & 42 & 13 & \\
\hline No & 49 & 29.5 & 0 & & 36 & 13 & \\
\hline Distant recurrence $^{\mathrm{a}}$ & & & & $0.087^{\mathrm{b}}$ & & & $0.651^{\mathrm{c}}$ \\
\hline Yes & 52 & 15.8 & 0 & & 40 & 12 & \\
\hline No & 52 & 30.1 & 0 & & 38 & 14 & \\
\hline Methylation $^{\mathrm{a}}$ & & & & $0.056^{\mathrm{b}}$ & & & $0.089^{c}$ \\
\hline Yes & 26 & 12.3 & 0 & & 23 & 3 & \\
\hline No & 41 & 21.6 & 0 & & 29 & 12 & \\
\hline $\mathrm{rs} 4430796^{\mathrm{a}}$ & & & & $0.993^{\mathrm{b}}$ & & & $0.535^{\mathrm{d}}$ \\
\hline Yes & 44 & 20.4 & 0 & & 35 & 9 & \\
\hline No & 21 & 13.9 & 0 & & 15 & 6 & \\
\hline rs $757210^{a}$ & & & & $0.613^{\mathrm{b}}$ & & & $0.476^{\mathrm{d}}$ \\
\hline Yes & 52 & 17.9 & 0 & & 41 & 11 & \\
\hline No & 13 & 19.8 & 0 & & 9 & 4 & \\
\hline rs7405776 ${ }^{a}$ & & & & $0.071^{\mathrm{b}}$ & & & $0.115^{\mathrm{d}}$ \\
\hline Yes & 44 & 24.8 & 0 & & 31 & 13 & \\
\hline No & 21 & 5.0 & 0 & & 19 & 2 & \\
\hline
\end{tabular}

${ }^{\mathrm{a} D a t a}$ not available for all cases; ${ }^{\mathrm{P}} \mathrm{P}-\mathrm{values}$ are based on Mann-Whitney U test or Kruskal-Wallis H-test; ${ }^{\mathrm{C}} \mathrm{P}$-values are based on Pearson $\chi^{2}$ test; ${ }^{\mathrm{d} P}$-values are based on Fisher exact test. The protein expression of HNF1B was categorized into group 1 (H-score 0-19) and group 2 (H-score 20-300).

potential influence on EMT. They found a significant association between the SNP rs757210 and tumour methylation of HNF1B in HGSC, but there was no significant association with HNF1B expression levels (5). Other authors, such as Shen et al, investigated selected SNPs in HGSC (rs7405776) and OCCC (rs11651755), and they did not prove any significant, but only borderline association between rs7405776 and increased promoter methylation in HGSC $(\mathrm{P}=0.07)(10)$. In accordance with those findings our results also showed no significant relationship between promoter methylation and rs7405776, or the other SNPs (rs4430796, and rs757210) which are most commonly implicated in carcinomas of the female genital system (Table III). Therefore, it is not surprising that we and others have also observed no association between the abovementioned SNPs and HNF1B protein expression or mRNA expression $(5,10)$.

We observed methylation of the HNF1B promoter region in $38.8 \%(26 / 67)$ HGSC, which is similar to the observations of other authors: $41.3 \%$ (12/29) (31), 42\% (120/286) (10), $45 \%$ (18/40) (32) and 50.8\% (31/61) (33). However, the TCGA data shows a higher frequency of methylated HGSCs $(55.4 \%$; $315 / 569)$. Our data showed no statistically significant correlation between the methylation status and any other tested characteristics, including the tumour stage (Table III), which is in concordance with other studies (31). Data from studies published by Bubancova et al, Baranova et al, and the TCGA 
Table II. Association of ECI2 protein expression (H-score) and clinico-pathological characteristics, based on 122 cases of HGSC.

\begin{tabular}{|c|c|c|c|c|c|c|c|}
\hline Characteristic & $\mathrm{N}$ & H-score mean & H-score median & $\mathrm{P}$-value & H-score group 1 & H-score group 2 & P-value \\
\hline $\begin{array}{l}\text { Age of diagnosis }(\text { mean }=59 \text {, } \\
\text { median }=60) \text {, years }\end{array}$ & & & & $0.673^{\mathrm{b}}$ & & & $0.508^{c}$ \\
\hline$<60$ & 59 & 110.1 & 105 & & 14 & 45 & \\
\hline$\geq 60$ & 63 & 104.7 & 105 & & 18 & 45 & \\
\hline $\mathrm{FIGO}^{\mathrm{a}}$ & & & & $0.372^{\mathrm{b}}$ & & & $0.242^{\mathrm{d}}$ \\
\hline I & 10 & 96.7 & 102 & & 4 & 6 & \\
\hline II & 7 & 119.5 & 120 & & 0 & 7 & \\
\hline III & 83 & 106.2 & 105 & & 21 & 62 & \\
\hline IV & 20 & 107.6 & 102 & & 7 & 13 & \\
\hline Lymphovascular invasion $^{a}$ & & & & $0.217^{\mathrm{b}}$ & & & $0.278^{\mathrm{b}, \mathrm{c}}$ \\
\hline Yes & 63 & 104.3 & 104 & & 18 & 45 & \\
\hline No & 28 & 116.1 & 114 & & 5 & 23 & \\
\hline Neoadjuvant therapy & & & & $0.901^{\mathrm{b}}$ & & & $0.511^{\mathrm{c}}$ \\
\hline Yes & 28 & 109.6 & 105 & & 6 & 22 & \\
\hline No & 94 & 106.6 & 105 & & 26 & 68 & \\
\hline Local recurrence $^{\mathrm{a}}$ & & & & $0.517^{\mathrm{b}}$ & & & $0.332^{\mathrm{c}}$ \\
\hline Yes & 55 & 107.5 & 104 & & 17 & 38 & \\
\hline No & 49 & 105.1 & 110 & & 11 & 38 & \\
\hline Distant recurrence ${ }^{c}$ & & & & $0.556^{\mathrm{b}}$ & & & $0.658^{\mathrm{c}}$ \\
\hline Yes & 52 & 111.0 & 107 & & 13 & 39 & \\
\hline No & 52 & 101.8 & 105 & & 15 & 37 & \\
\hline
\end{tabular}

${ }^{\mathrm{a} D a t a}$ not available for all cases; ${ }^{\mathrm{b}} \mathrm{P}-\mathrm{values}$ are based on Mann-Whitney U test or Kruskal-Wallis H-test; ${ }^{\mathrm{C}} \mathrm{P}$-values are based on Pearson $\chi^{2}$ test; ${ }^{d} \mathrm{P}$-values are based on Fisher's exact test. The protein expression of ECI2 was categorized into group 1 (H-score 0-99) and group 2 (H-score 100-300).

data showed a more frequent HNF1B promoter methylation in tumours of a later stage (stage I/II 36.2 vs. stage III/IV $60.1 \% ; 28.6$ vs. $57.5 \% ; 34.0$ vs. $57.3 \%$ ). We observed the same trend (stage I/II 30 vs. stage III/IV 41.7\%), but this finding was not statistically significant $(\mathrm{P}>0.05)$ in our cohort.

The mutation analysis revealed that in one case of the 61 (1.6\%) there was a novel somatic $H N F 1 B$ truncating mutation in exon 5 (p.Q355X). This pathogenic variant is predicted to encode a truncated non-functional protein, which is in accordance with immunohistochemical analysis where complete negativity of HNF1B staining $(\mathrm{H}$-score $=0)$ was observed. Our findings support our previous assumption that the expression of HNF1B in tumour tissue is downregulated by either hypermethylation of the promoter, or by other potential regulatory mechanism which affects transcriptional activation, rather than mutations in the HNF1B coding sequence or posttranscriptional or posttranslational modifications of gene expression (22).

We also analysed the immunohistochemical and mRNA expression of HNF1B, with the aim of exploring whether there is a correlation between the expression and clinico-pathological characteristics. In total, we found immunohistochemical positivity of HNF1B in 23\% (28/122) of cases and discovered a significant association between higher values of the $\mathrm{H}$-score and lymphovascular invasion $(\mathrm{P}=0.025)$. This finding supports the contention of some authors that HNF1B induces transformation and epithelial-to-mesenchymal transition, which can contribute to the acquisition of the invasive properties which are essential for the metastasis's invasive behaviour $(6,34)$. No associations between HNF1B or ECI2 protein expression and disease outcome (Fig. 2) were observed.

So far, studies concerning the expression of HNF1B in HGSC have been focused on the diagnosis of OCCC and the differentiation of tumours with clear cell morphological features from clear cell carcinomas (15,35-37). The discriminative threshold in these studies was adjusted to differentiate OCCC from other tumour types and, as such, HNF1B expression was confirmed as a specific marker for the diagnosis of OCCC. Huang et al found positive expression of HNF1B in only $2.9 \%$ (1/35 cases) of HGSCs (35). However, only tumours with diffuse, and moderate or strong nuclear positivity were regarded as positive by these authors, which is quite a rare occurrence in HGSC. In their study, Kao et al investigated HGSCs with clear cell changes and found that only 5\% (3/60 cases) reached an $\mathrm{H}$-score of $>10$ (this H-score threshold was determined as the cut-off to differentiate HGSC and endometroid ovarian cancer from OCCC in their study) (15). Kobel et al investigated the expression of HNF1B in 133 OCCCs and 200 HGSCs, and found focally distributed nuclear positivity in $4.8 \%$ of HGSCs (36). In another study, Li et al reported HNF1B positivity in $13.3 \%$ (4/30 cases) of HGSCs (37), and none of the HGSC cases showed strong nuclear positivity. The rather lower reported positivity when 
Table III. Association of HNF1B promoter methylation and selected clinicopathological characteristics, based on 67 cases of HGSC. P-values are based on Pearson $\chi^{2}$ test.

\begin{tabular}{|c|c|c|c|}
\hline Characteristics & Methylation yes & Methylation no & P-value \\
\hline Age of diagnosis, years & & & 0.307 \\
\hline$<60$ & 16 & 20 & \\
\hline$\geq 60$ & 10 & 21 & \\
\hline $\mathrm{FIGO}^{\mathrm{a}}$ & & & 0.714 \\
\hline I & 1 & 4 & \\
\hline II & 2 & 3 & \\
\hline III & 17 & 27 & \\
\hline IV & 6 & 6 & \\
\hline Lymphovascular invasion $^{\mathrm{a}}$ & & & 0.778 \\
\hline Yes & 12 & 26 & \\
\hline No & 5 & 9 & \\
\hline Neoadjuvant therapy & & & 0.478 \\
\hline Yes & 5 & 11 & \\
\hline No & 21 & 30 & \\
\hline Local recurrence ${ }^{\mathrm{a}}$ & & & 0.252 \\
\hline Yes & 14 & 19 & \\
\hline No & 6 & 16 & \\
\hline Distant recurrence ${ }^{\mathrm{a}}$ & & & 0.878 \\
\hline Yes & 11 & 20 & \\
\hline No & 9 & 15 & \\
\hline $\mathrm{rs} 4430796^{\mathrm{a}}$ & & & 0.615 \\
\hline Yes & 16 & 28 & \\
\hline No & 9 & 12 & \\
\hline $\operatorname{rs} 757210^{a}$ & & & 0.524 \\
\hline Yes & 19 & 33 & \\
\hline No & 6 & 7 & \\
\hline rs7405776 ${ }^{\mathrm{a}}$ & & & 0.614 \\
\hline Yes & 16 & 28 & \\
\hline No & 9 & 12 & \\
\hline
\end{tabular}

${ }^{\mathrm{a} D a t a}$ not available for all cases.

compared to our results can most likely be explained by differences in methodology, such as the use of a different antibody with different pretreatment, or different visualization methods, as well as different criteria for the evaluation of HNF1B expression.

Our data showed a statistically significant positive correlation between mRNA and protein expression of HNF1B $(\mathrm{P}=0.001)$. This finding implies that the regulation of HNF1B expression on the translational or posttranslational level probably does not play a significant role.

The data concerning the significance of ECI2 in ovarian carcinomas, including HGSC, is currently unknown. According to studies on prostate cancer, the inhibition of ECI2 can trigger acute metabolic stress of the tumour cells, and thus targeting ECI 2 could be potentially used as a therapeutic approach (19). According to some studies, a higher expression of the ECI 2 protein is associated with a poor outcome in prostate cancer, but the data concerning the expression of
ECI2 in ovarian cancer is lacking (18). In our study, we found cytoplasmic expression of ECI2 in all but 7 cases (H-score up to 190). Our data showed no correlation between any of the clinico-pathological characteristics and survival on a statistically significant level (Table II). Regarding the connection between the expression of HNF1B and ECI2, our data showed a significant positive relationship on a protein level $(\mathrm{P}=0.005)$, but we did not observe correlation at an mRNA level. However, this is in concordance with the re-analysis of the TGCA dataset, where no correlation between the mRNA expression of HNF1B/ECI2 has been found.

In conclusion, the comprehensive analysis of molecular and immunohistochemical characteristics of HNF1B and its possible downstream target ECI2 brought about several important findings. Our data from the expression analysis confirmed a generally low HNF1B expression on a protein level, which positively correlated with lymphovascular invasion, but with no other clinico-pathological characteristics. 

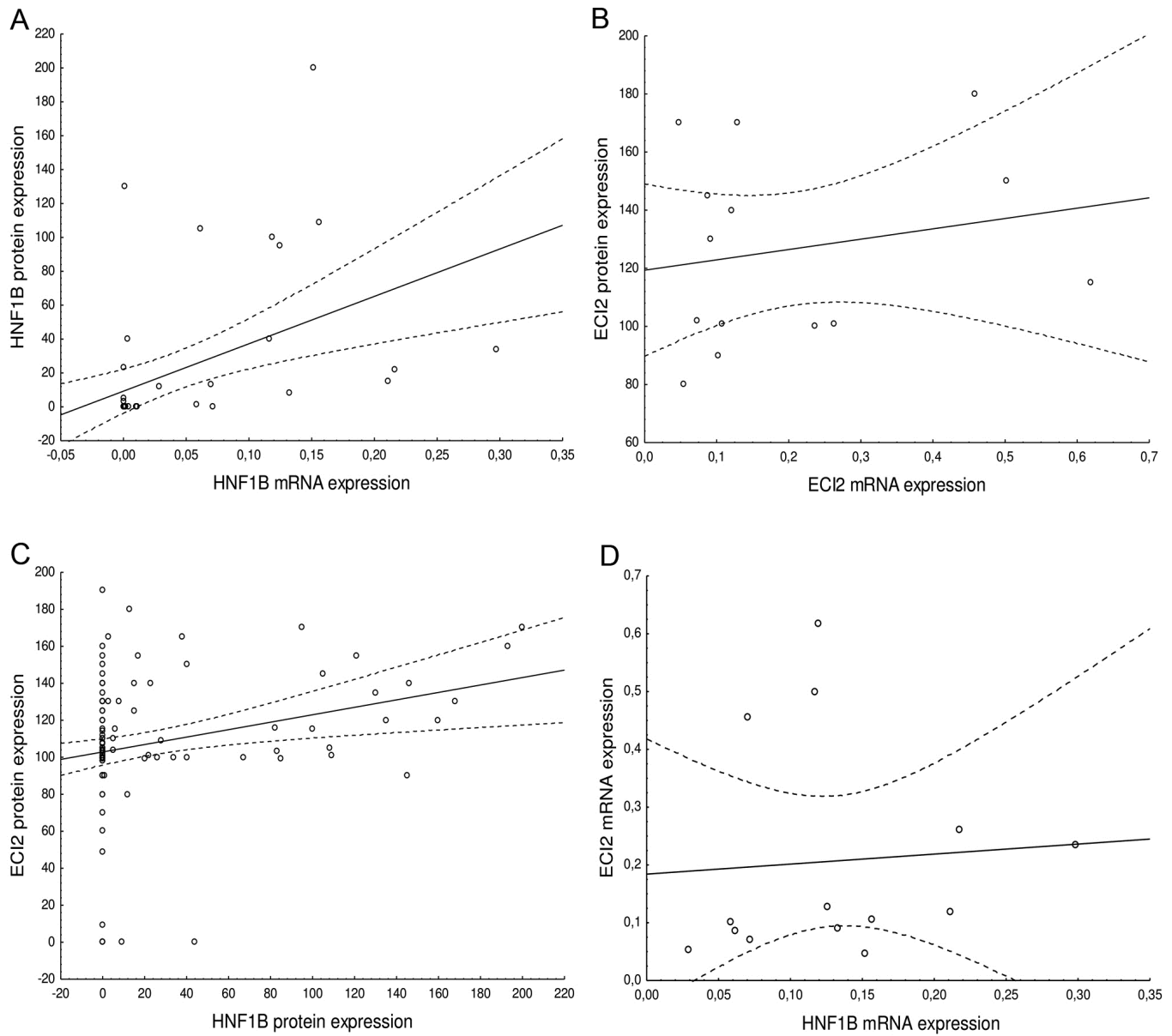

Figure 3. Association of mRNA expression and protein expression of (A) HNF1B. $\left(F=12.18, R^{2}=0.213, N=47, P=0.001\right)$ and (B) $E C I 2\left(F=0.51, R^{2}=0.041, N=14\right.$, $\mathrm{P}=0.488$ ), and the association of expression of HNF1B and $\mathrm{ECI} 2$ on a $(\mathrm{C})$ protein level ( $\left.\mathrm{F}=8.03, \mathrm{R}^{2}=0.063, \mathrm{~N}=122, \mathrm{P}=0.005\right)$ and (D) mRNA level (F=0.05, $\mathrm{R}^{2}=0.005, \mathrm{~N}=14, \mathrm{P}=0.816$ ). Dashed lines represent 0.95 confidence interval. HNF1B, hepatocyte nuclear factor 1 $\beta$; ECI2, enoyl-CoA $(\Delta)$ isomerase 2

Nevertheless, despite the observed low levels, the expression of HNF1B was noted in 28/122 cases (23\%), which supports the fact that the specificity of HNF1B as a marker of clear cell carcinoma is relatively low. However, no case of HGSC in our study reached a $\mathrm{H}$-score higher than 200. Taking the extent of expression into account, the sensitivity of HNF1B is significantly higher, given that in CCC the expression of HNF1B is commonly strong and diffuse. Furthermore, we observed a statistically significant correlation between the mRNA and protein expression of HNF1B, which suggests that posttranscriptional and posttranslational mechanisms are probably not involved in the regulation of the $H N F 1 B$ gene expression in HGSC.

This is the first time that protein and mRNA expression of ECI2, one of the possible HNF1B downstream targets, has been analysed in HGSC. Our data shows that a low level of immunohistochemical expression was present in the majority of HGSCs. We have found a significant positive relationship between HNF1B and ECI2 on a protein level, which is in accordance with the previous findings in prostate cancer (19). Interestingly, the expression of HNF1B and ECI2 on an mRNA level did not show a positive association, which is supported by the re-analysis of the TCGA dataset. However, no correlation between any of the clinico-pathological characteristics or survival analyses and ECI2 was found.
Therefore, ECI2 does not seem to be an eligible prognostic marker for HGSC.

\section{Acknowledgements}

The authors would like to thank Assistant Professor Zachary H.K. Kendall, B.A. (Institute for History of Medicine and Foreign Languages, First Faculty of Medicine, Charles University in Prague) for the English language editing.

\section{Funding}

This work was supported by Ministry of Health, Czech Republic (research project AZV 17-28404A and conceptual development of research organization 64165; General University Hospital in Prague), by Charles University (Project Progress Q28/LF1 and SVV 260367), and by European Regional Development Fund (project EF16_013/0001674, BBMRI_CZ LM2018125, and OPPK-Research Laboratory of Tumor Diseases, CZ.2.16/3.1.00/24509).

\section{Availability of data and materials}

The datasets used and/or analysed during the current study are available from the corresponding author on reasonable request. 


\section{Authors' contributions}

Study concept and design: KN and PD. Samples and clinical data collection: DC, KJ, MB, HQB. Preparation and analysis of samples: KN, MB, NH, JH and EK. Statistical analyses and data interpretation: RM, IS, KN. Drafting of the manuscript: KN, MB, JH, NH, IS, PD, RM, DC and KJ. Proofread the manuscript: IS, PD and KN. KN and PD confirmed the authenticity of all the raw data. All authors read and approved the final manuscript.

\section{Ethics approval and consent to participate}

The present study was approved by the Ethics Committee of the General University Hospital in Prague in compliance with the Helsinki Declaration (ethical approval number 41/16 Grant VES 2017 AZV VFN). The requirement for written informed consent was waived due to the retrospective nature of the study.

\section{Patient consent for publication}

Not applicable.

\section{Competing interests}

The authors declare that they have no competing interests.

\section{References}

1. Liu Y, Kanyomse Q and Xie Y: Tumor-suppressive activity of Hnf1 $\beta$ in Wilms' tumor. Biosci Biotechnol Biochem 83: 2008-2015, 2019.

2. Suzuki E, Kajita S, Takahashi H, Matsumoto T, Tsuruta T and Saegusa M: Transcriptional upregulation of HNF-1 $\beta$ by NF-KB in ovarian clear cell carcinoma modulates susceptibility to apoptosis through alteration in bcl-2 expression. Lab Invest 95: 962-972, 2015

3. Tsuchiya A, Sakamoto M, Yasuda J, Chuma M, Ohta T, Ohki M, Yasugi T, Taketani Y and Hirohashi S: Expression profiling in ovarian clear cell carcinoma: Identification of hepatocyte nuclear factor-1 beta as a molecular marker and a possible molecular target for therapy of ovarian clear cell carcinoma. Am J Pathol 163: 2503-2512, 2003

4. Bartu M, Dundr P, Nemejcova K, Ticha I, Hojny H and Hajkova N: The role of HNF1B in tumorigenesis of solid tumours: A review of current knowledge. Folia Biol (Praha) 64: 71-83, 2018.

5. Ross-Adams H, Ball S, Lawrenson K, Halim S, Russell R, Wells C, Strand SH, Ørntoft TF, Larson M, Armasu S, et al HNF1B variants associate with promoter methylation and regulate gene networks activated in prostate and ovarian cancer. Oncotarget 7: 74734-74746, 2016.

6. Matsui A, Fujimoto J, Ishikawa K, Ito E, Goshima N, Watanabe S and Semba K: Hepatocyte nuclear factor 1 beta induces transformation and epithelial-to-mesenchymal transition. FEBS Lett 590: 1211-1221, 2016.

7. Kato N, Sasou S and Motoyama T: Expression of hepatocyte nuclear factor-1beta (HNF-1beta) in clear cell tumors and endometriosis of the ovary. Mod Pathol 19: 83-89, 2006.

8. Mandato VD, Farnetti E, Torricelli F, Abrate M, Casali B, Ciarlini G, Pirillo D, Gelli MC, Nicoli D, Grassi M, et al: HNF1B polymorphism influences the prognosis of endometrial cancer patients: A cohort study. BMC Cancer 15: 229, 2015.

9. Setiawan VW, Haessler J, Schumacher F, Cote ML, Deelman E, Fesinmeyer MD, Henderson BE, Jackson RD, Vöckler JS, Wilkens LR, et al: HNF1B and endometrial cancer risk: Results from the PAGE study. PLoS One 7: e30390, 2012.

10. Shen H, Fridley BL, Song H, Lawrenson K, Cunningham JM, Ramus SJ, Cicek MS, Tyrer J, Stram D, Larson MC, et al: Epigenetic analysis leads to identification of HNF1B as a subtype-specific susceptibility gene for ovarian cancer. Nat Commun 4: 1628, 2013.
11. Spurdle AB, Thompson DJ, Ahmed S, Ferguson K, Healey CS O'Mara T, Walker LC, Montgomery SB, Dermitzakis ET; Australian National Endometrial Cancer Study Group, et al: Genome-wide association study identifies a common variant associated with risk of endometrial cancer. Nat Genet 43: 451-454, 2011.

12. Kato $\mathrm{N}$ and Motoyama T: Hepatocyte nuclear factor-1beta (HNF-1beta) in human urogenital organs: Its expression and role in embryogenesis and tumorigenesis. Histol Histopathol 24: 1479-1486, 2009.

13. Yamamoto S, Tsuda H, Aida S, Shimazaki H, Tamai S and Matsubara O: Immunohistochemical detection of hepatocyte nuclear factor 1beta in ovarian and endometrial clear-cell adenocarcinomas and nonneoplastic endometrium. Hum Pathol 38: 1074-1080, 2007.

14. Fadare O and Liang SX: Diagnostic utility of hepatocyte nuclear factor 1-beta immunoreactivity in endometrial carcinomas: Lack of specificity for endometrial clear cell carcinoma. Appl Immunohistochem Mol Morphol 20: 580-587, 2012.

15. Kao YC, Lin MC, Lin WC, Jeng YM and Mao TL: Utility of hepatocyte nuclear factor-1 $\beta$ as a diagnostic marker in ovarian carcinomas with clear cells. Histopathology 61: 760-768, 2012.

16. Nemejcova K, Ticha I, Kleiblova P, Bártů M, Cibula D, Jirsová K and Dundr P: Expression, epigenetic and genetic changes of HNF1B in endometrial lesions. Pathol Oncol Res 22: 523-530, 2016.

17. Dan C, Zhang H, Zeng W, Huang L, Gong X, Li H, Yang E, Wang L and Yao Q: HNF1B expression regulates ECI2 gene expression, potentially serving a role in prostate cancer progression. Oncol Lett 17: 1094-1100, 2019.

18. Houten SM, Violante S, Ventura FV and Wanders RJ: The biochemistry and physiology of mitochondrial fatty acid $\beta$-oxidation and its genetic disorders. Annu Rev Physiol 78: 23-44, 2016.

19. Itkonen HM, Brown M, Urbanucci A, Tredwell G, Ho Lau C, Barfeld S, Hart C, Guldvik IJ, Takhar M, Heemers HV, et al: Lipid degradation promotes prostate cancer cell survival. Oncotarget 8: 38264-38275, 2017.

20. Fan J, Li X, Issop L, Culty $M$ and Papadopoulos V: ACBD2/ECI2-mediated peroxisome-mitochondria interactions in leydig cell steroid biosynthesis. Mol Endocrinol 30: 763-782, 2016.

21. Hirsch FR, Dziadziuszko R, Thatcher N, Mann H, Watkins C, Parums DV, Speake G, Holloway B, Bunn PA Jr and Franklin WA: Epidermal growth factor receptor immunohistochemistry: Comparison of antibodies and cutoff points to predict benefit from gefitinib in a phase 3 placebo-controlled study in advanced nonsmall-cell lung cancer. Cancer 112: 1114-1121, 2008.

22. Bartu M, Hojny J, Hajkova N, Michálková R, Krkavcová E, Simon K, Frýba V, Stružinská I, Němejcová K and Dundr P: Expression, epigenetic, and genetic changes of HNF1B in colorectal lesions: An analysis of 145 cases. Pathol Oncol Res 26: 2337-2350, 2020

23. DundrP,Bartu M,Hojny J,Michálková R, HájkováN, Stružinská I, Krkavcová E, Hadravský L, Kleissnerová L, Kopejsková J, et al: HNF1B, EZH2 and ECI2 in prostate carcinoma. Molecular, immunohistochemical and clinico-pathological study. Sci Rep 10: 14365, 2020.

24. Kim HY: Statistical notes for clinical researchers: Chi-squared test and Fisher's exact test. Restor Dent Endod 42: 152-155, 2017.

25. Cancer Genome Atlas Research Network: The molecular taxonomy of primary prostate cancer. Cell 163: 1011-1025, 2015.

26. Kato N, Tamura G and Motoyama T: Hypomethylation of hepatocyte nuclear factor-1beta (HNF-1beta) CpG island in clear cell carcinoma of the ovary. Virchows Arch 452: 175-180, 2008.

27. Lau HH, Ng NHJ, Loo LSW, Jasmen JB and Teo AKK: The molecular functions of hepatocyte nuclear factors-In and beyond the liver. J Hepatol 68: 1033-1048, 2018.

28. Kalloger SE, Kobel M, Leung S, Mehl E, Gao D, Marcon KM, Chow C, Clarke BA, Huntsman DG and Gilks CB: Calculator for ovarian carcinoma subtype prediction. Mod Pathol 24: 512-521, 2011.

29. Tomassetti A, De Santis G, Castellano G, Miotti S, Mazzi M, Tomasoni D, Van Roy F, Carcangiu ML and Canevari S: Variant HNF1 modulates epithelial plasticity of normal and transformed ovary cells. Neoplasia 10: 1481-1492, 2008.

30. Bowtell DD, Bohm S, Ahmed AA, Aspuria PJ, Bast RC Jr, Beral V, Berek JS, Birrer MJ, Blagden S, Bookman MA, et al: Rethinking ovarian cancer II: Reducing mortality from high-grade serous ovarian cancer. Nat Rev Cancer 15: 668-679, 2015. 
31. Terasawa K, Toyota M, Sagae S, Ogi K, Suzuki H, Sonoda T, Akino K, Maruyama R, Nishikawa N, Imai K, et al: Epigenetic inactivation of TCF2 in ovarian cancer and various cancer cell lines. Br J Cancer 94: 914-921, 2006.

32. Bubancova I, Kovarikova H, Laco J, Ruszova E, Dvorak O, Palicka V and Chmelarova M: Next-generation sequencing approach in methylation analysis of HNF1B and GATA4 genes: Searching for biomarkers in ovarian cancer. Int J Mol Sci 18: 474, 2017.

33. Baranova I, Kovarikova H, Laco J, Sedlakova I, Vrbacky F, Kovarik D, Hejna P, Palicka V and Chmelarova M: Identification of a four-gene methylation biomarker panel in high-grade serous ovarian carcinoma. Clin Chem Lab Med 58: 1332-1340, 2020.

34. Chaffer CL, San Juan BP, Lim E and Weinberg RA: EMT, cell plasticity and metastasis. Cancer Metastasis Rev 35: 645-654, 2016.

35. Huang W, Cheng X, Ji J, Zhang J and Li Q: The application value of HNF-1 $\beta$ transcription factor in the diagnosis of ovarian clear cell carcinoma. Int J Gynecol Pathol 35: 66-71, 2016.
36. Kobel M, Kalloger SE, Carrick J, Huntsman D, Asad H, Oliva E, Ewanowich CA, Soslow RA and Gilks CB: A limited panel of immunomarkers can reliably distinguish between clear cell and high-grade serous carcinoma of the ovary. Am J Surg Pathol 33: 14-21, 2009.

37. Li Q, Zeng X, Cheng X, Zhang J, Ji J, Wang J, Xiong K, Qi Q and Huang W: Diagnostic value of dual detection of hepatocyte nuclear factor 1 beta (HNF-1 $\beta$ ) and napsin A for diagnosing ovarian clear cell carcinoma. Int J Clin Exp Pathol 8: 8305-8310, 2015.

This work is licensed under a Creative Commons Attribution-NonCommercial-NoDerivatives 4.0 International (CC BY-NC-ND 4.0) License. 